\title{
Image of the month: A misleading chest X-ray - not all opacification is effusion
}

\author{
Authors: Ioannis Psallidas, ${ }^{A}$ John P Corcoran, ${ }^{B}$ Robert J Hallifax ${ }^{C}$ and Najib M Rahman ${ }^{D}$
}

Pleural effusions are a common problem in both general and respiratory medicine, with pleural fluid aspiration and chest tube insertion being among the most frequently performed medical procedures. Although guidelines advocate thoracic ultrasound in the diagnostic evaluation of pleural effusion, its use is not yet fully established in everyday clinical practice. ${ }^{1}$ However, a diagnosis of pleural effusion based on only radiographic and physical examination may be misleading and can result in iatrogenic complications. ${ }^{2,3}$

A 69-year-old man was referred for evaluation of a left-sided pleural effusion. His symptoms included dyspnoea, which had worsened over 6 months, dysphagia and coughing after eating. His chest X-ray (Fig 1) showed a left-sided pleural effusion, mediastinal shift to the right and loculated air in the left mid-zone, which was suspected to be a hydropneumothorax and pleural infection. However, thoracic ultrasound (Fig 2) identified only bowel contents in the left hemithorax with no evidence of pleural effusion. Subsequent computed tomography (Figs 3 and 4) confirmed these findings with evidence of a large left diaphragmatic hernia, bowel and omentum occupying approximately $70 \%$ of the left hemithorax, and partial collapse of the left main bronchus due to extrinsic compression. The patient was referred to thoracic surgery for replacement of the hernia and hemidiaphragmatic reconstruction.

This case reinforces the importance of thoracic ultrasound in the evaluation of suspected pleural effusion as a straightforward, non-invasive and non-irradiating diagnostic technique. Current evidence recommend that ultrasound guided pleural aspirations are associated with lower complications, particularly pneumothoraces and inadvertent organ punctures. ${ }^{4}$ Current training of respiratory physicians should include the minimum standard requirements from the Royal College of Radiologists to achieve level 1 competency in

Authors: ${ }^{\text {A }}$ clinical and research fellow, Oxford Centre for Respiratory Medicine Oxford University Hospitals NHS Trust, Oxford, UK; ${ }^{B}$ Clinical and research fellow, Oxford Centre for Respiratory Medicine, Oxford University Hospitals NHS Trust, Oxford, UK; ${ }^{C}$ Clinical and research fellow, Oxford Centre for Respiratory Medicine, Oxford University Hospitals NHS Trust, Oxford, UK; D consultant and senior lecturer, Oxford Centre for Respiratory Medicine, Oxford University Hospitals NHS Trust, Oxford, UK

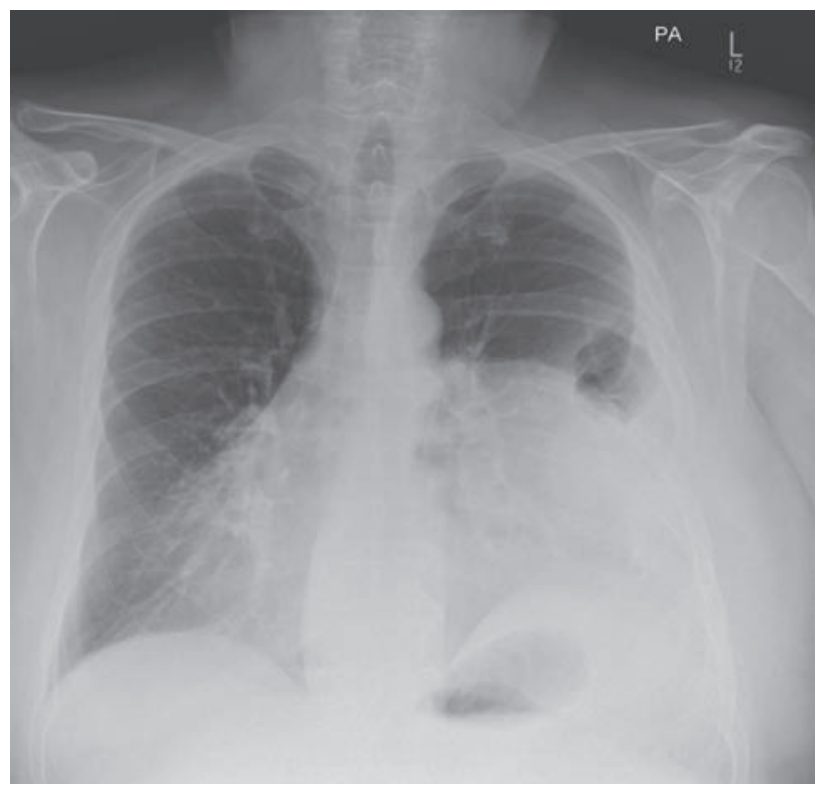

Fig 1. Chest X-ray with suspected left pleural effusion.

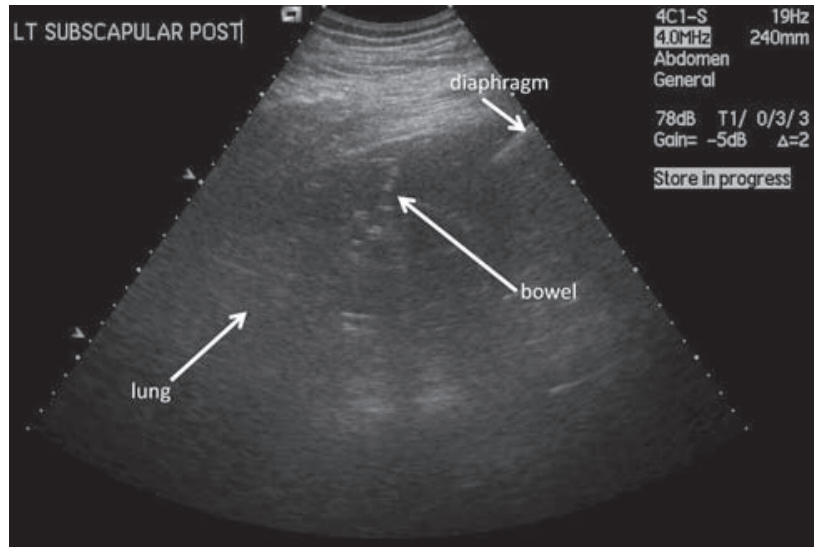

Fig 2. Ultrasound (posterior chest, 4th ICS), showing bowel (mixed echogenicity above diaphragm) with underlying compressed lung. ICS = intercostal space. 


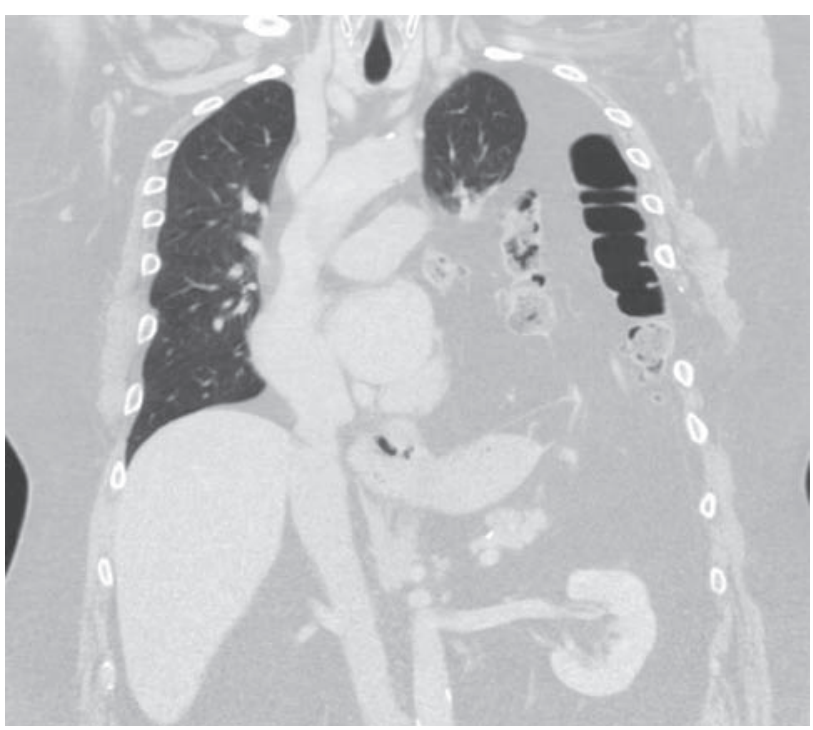

Fig 3. Coronal computed tomography showing abdominal contents extending into left hemithorax.

ultrasound imaging. The increased use of this technique after appropriate training will gradually maximise the potential benefits for patients in the scope of diagnosis and management of pleural diseases.

\section{Acknowledgement}

Dr Ioannis Psallidas is the recipient of a European Respiratory Society Fellowship (LTRF 2013-1824).

\section{References}

1 Hooper C, Lee YCG, Maskell N; BTS Pleural Guideline Group. Investigation of a unilateral pleural effusion in adults: British Thoracic Society Pleural Disease Guideline 2010. Thorax 2010;65(Suppl 2):ii4-17.

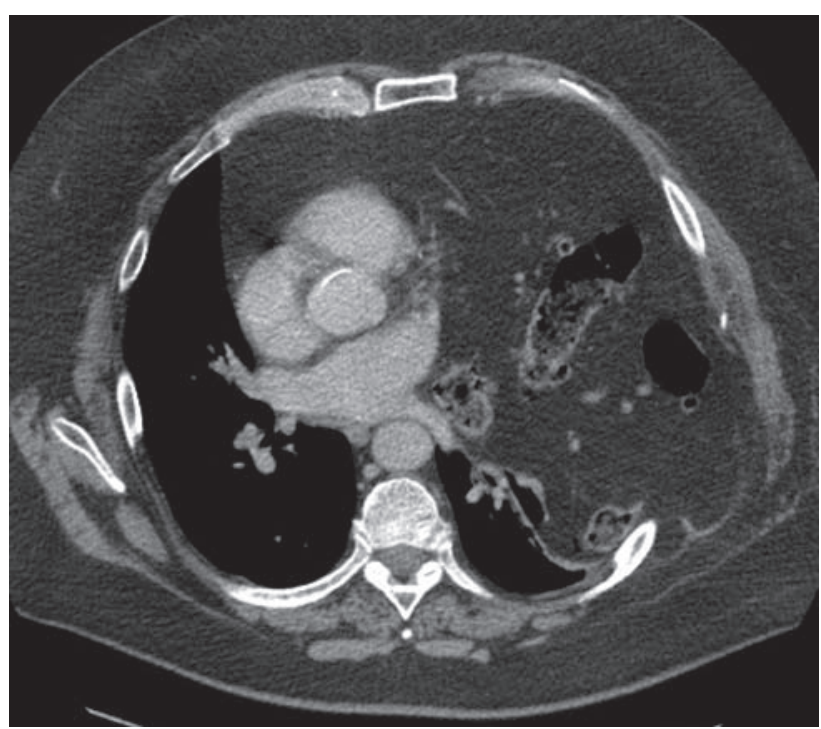

Fig 4. Axial computed tomography showing mediastinal shift and left-sided rib splaying caused by herniation of abdominal contents.

2 Duncan DR, Morgenthalen TI, Ryu JH, Daniels CE. Reducing iatrogenic risk in thoracentesis: establishing best practice via experiential training in a zero-risk environment. Chest 2009;135:1315-20.

3 Diacon AH, Brutsche MH, Solèr M. Accuracy of pleural puncture sites: a prospective comparison of clinical examination with ultrasound. Chest 2003;123:436-41.

4 Havelock T, Teoh R, Laws D, Gleeson F; BTS Pleural Disease Guideline Group. Pleural procedures and thoracic ultrasound: British Thoracic Society Pleural Disease Guideline 2010. Thorax 2010;65(Suppl 2):ii61-76.

Address for correspondence: Dr I Psallidas, Oxford Centre for Respiratory Medicine, Oxford University Hospitals NHS Trust, Churchill Hospital Old Road, Oxford OX3 7LE.

Email: ioannis.psallidas@ouh.nhs.uk 